\author{
Angelos Kyriacou $^{1 \star}$, V Lorelei Salazar ${ }^{2}$ \\ and Sunethra Ghattamaneni ${ }^{3}$ \\ ${ }^{I}$ Department of Endocrinology, U.K \\ ${ }^{2}$ Department of Radiology, U.K \\ ${ }^{3}$ Salford Royal NHS Foundation Trust, Salford, \\ Greater Manchester, U.K \\ Dates: Received: 28 August, 2015; Accepted: 10 \\ October, 2015; Published: 14 October, 2015 \\ ${ }^{*}$ Corresponding author: Angelos Kyriacou, \\ Department of Endocrinology, U.K, E-mail: \\ angelos5@doctors.org.uk

\section{Case Report \\ Lymph Nodes Close to the Thyroid Isthmus Can Masquerade as Malignant Thyroid Nodules in Chronic Lymphocytic Thyroiditis}

www.peertechz.com

Keywords: Chronic lymphocytic thyroiditis; Lymph nodes; Thyroid nodules; Thyroid cancer

ISSN: 2640-7582

\section{Case Report}

A 40 year old lady who was four months postpartum, presented with a neck swelling and clinical and biochemical features consistent with postpartum thyroiditis. She had a family history of autoimmune thyroid disease. She underwent an ultrasound which showed features of diffuse thyroiditis and some nodules in the isthmus. An ultrasound-guided fine needle aspiration (FNA) biopsy of an isthmus nodule was undertaken which was reported as a follicular neoplasm (Thy 3f; corresponding Bethesda classification 4). Given that Thy 3 cytology carries a risk of malignancy at $9.5-40 \%$ and in accordance to the British Thyroid Association guidelines she was listed for diagnostic isthmusectomy [1]. The patient was referred to our hospital for a second opinion. At the time of review, she had been on thyroxine replacement therapy for approximately one month and was clinically and biochemically euthyroid and the anterior neck swelling had clinically improved. Thyroid peroxidase (TPO) antibodies were negative. A thyroid ultrasound was repeated which showed a diffusely and markedly hypoechoic, heterogeneous thyroid gland with a lobular outline that was consistent with chronic lymphocytic thyroiditis (CLT) (Figures 1-3). There were three small benignappearing pretracheal lymph nodes and several others inferior to the lower pole of the thyroid gland (level 6). Indeed, the isthmus 'thyroid nodule' that was previously biopsied was noted to be the extrathyroidal isthmus lymph nodes in close proximity to the thyroid. The patient was subsequently discussed in a thyroid nodule and cancer regional multidisciplinary team (MDT) meeting. A joint decision was taken by the MDT that surgery was unwarranted, but that the patient ought to be actively monitored clinically and radio logically. Indeed,

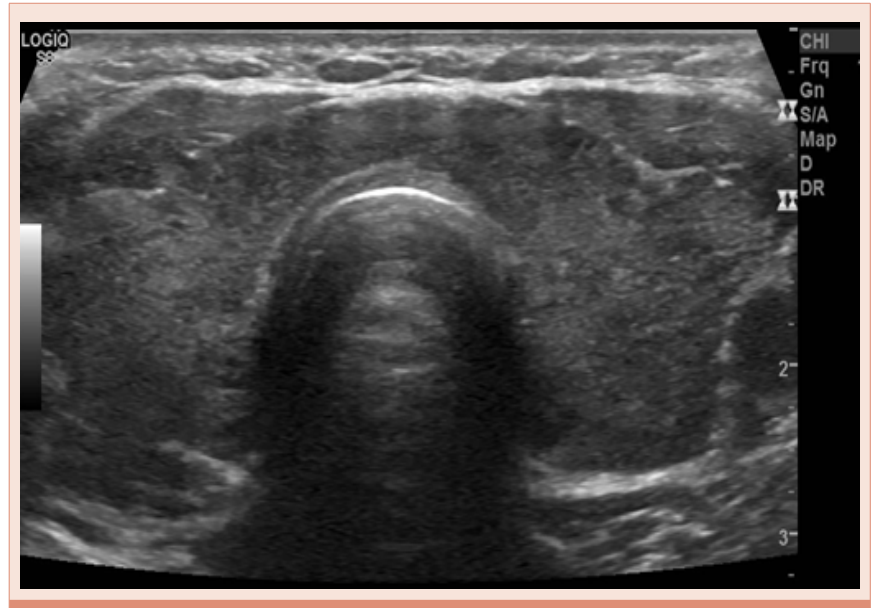

Figure 1: Transverse image of the thyroid gland indicating diffuse and prominent hypoechoic and heterogeneous appearances coupled with some enlargement of the gland, in keeping with chronic lymphocytic thyroiditis.

a follow-up ultrasound scan about seven months after her original presentation to our services revealed on-going appearances of CLT with persistence of small reactive-looking lymph nodes in close proximity to the thyroid gland (Figure 4). Again we could not identify any concerning thyroid nodules or any other sonographic features suggestive of malignancy and hence no FNA was required.

\section{Discussion}

Given that we could not identify any thyroid nodules in the 


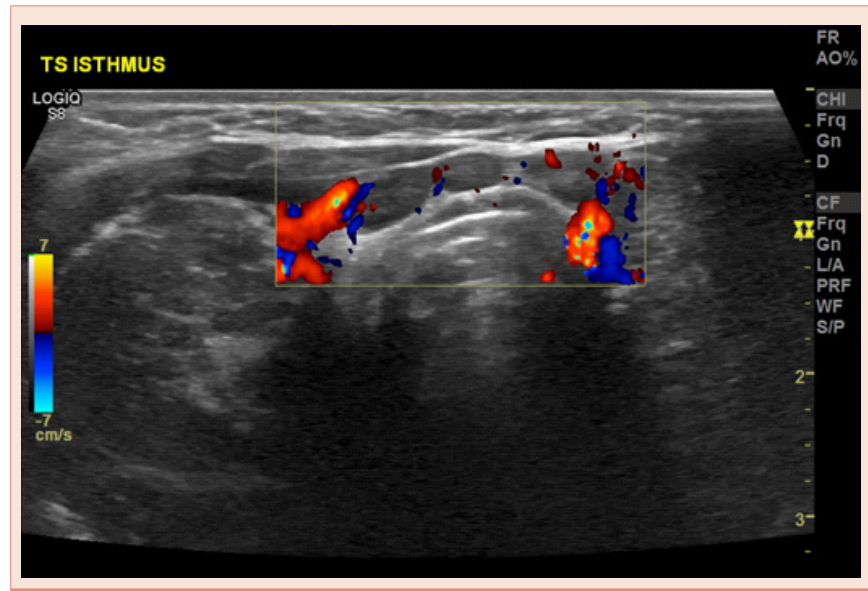

Figure 2: Transverse image of the thyroid gland indicating small, oval and hypoechoic lymph nodes with no abnormal vascularity on colour Doppler These are in close proximity to the thyroid isthmus and in keeping with reactive (benign) lymph nodes. Reactive pretracheal and paratracheal lymph nodes commonly occur in association with a chronic lymphocytic thyroiditis.

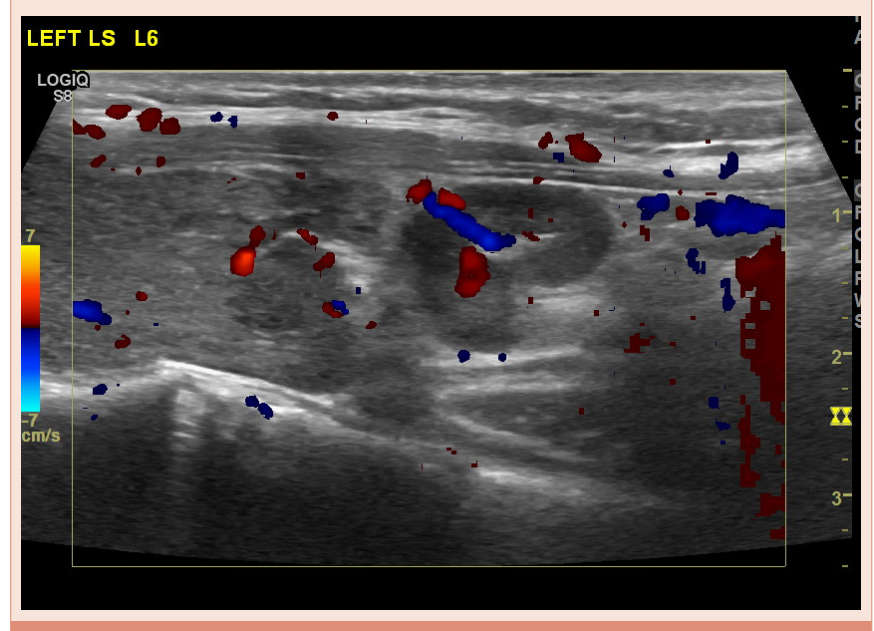

Figure 3: Longitudinal scan at the region of level 6 . Close interrogation of one of the lymph nodes demonstrates an echogenic hilum with hilar vascularity, a short-to-long axis ratio of less than 0.5 and absence of microcalcifications or any other concerning features, indicative that this is a benign lymph node.

isthmus area (or elsewhere), it is almost certain that because of the close proximity of the lymph nodes to the isthmus in an acutely inflamed gland, these were originally mistaken for thyroid nodules and targeted for FNA; the fact that the FNA result was reported as showing a follicular lesion indicates that some material has been obtained from the thyroid parenchyma (because of its adjacency to the lymph nodes) and was misinterpreted because it was thought to originate from a thyroid nodule.

Sonographic features of chronic lymphocytic thyroiditis (CLT) are well recognised and include the following: heterogeneously hypoechoic gland with a pseudomicronodular ('swiss cheese' or 'honeycomb') or pseudomacronodular pattern and ocassionally profoundly hypoechoic appearances without nodules [2,3]. Late in the natural history of CLT there may be fibrous replacement of the gland that can be reflected sonographically as hyperechoic and heterogeneous or, even speckled appearances and, ultimately, a small atrophic and hypovascular gland [2,3] and biochemically by the development of overt hypothyroidism. Pseudonodular appearances may be confused in 'less experienced hands' with a multi-nodular goitre $[2,3]$ but other sonographic features together with the clinical picture should guide to the correct impression. Diffuse heterogeneity, cyst with colloid clot, spongiform configuration and 'giraffe pattern' have previously been reported to confer $100 \%$ specificity for benign nodules in the landmark article by Bonavita et al. [4], and biopsyavoidance is recommended when these features are present. Reactive lymph nodes are almost universally found in CLT; they are commonly found at levels III and IV [5], as well as in the pre- and para-tracheal region [6]. Given the higher prevalence of papillary thyroid cancer in CLT, such lymph nodes need to be distinguished from malignant lymph nodes using the same sonographic criteria as per patients without CLT $[2,3,5]$. Features such as presence of an echogenic hilum, oval shape with a short-to-long axis ratio of less than 0.5, hilar vascularity (as opposed to peripheral or chaotic vascularity), and absence of calcifications and cystic necrosis are consistent with benignity.

This case emphasizes the need to be well aware of sonographic aspects of CLT and always correlate the clinical, biochemical and radiological characteristics in order to diagnose CLT. Reactive lymph nodes in close proximity to the isthmus are common in CLT and can easily be mistaken for thyroid nodules, potentially leading to unnecessary investigations and treatment, and patient anxiety. A limitation to our study is that the FNA was not repeated but given the lack of any suspicious features on sonography when this patient presented to ourselves and on follow-up imaging, the consensus reached by our very experienced MDT, and patient preference factors, we believe that further FNA could not be justified.

We propose that whenever uncertainty exists one approach is to radiologically follow the lesion in question. This allows for assessment of development of any new concerning morphological

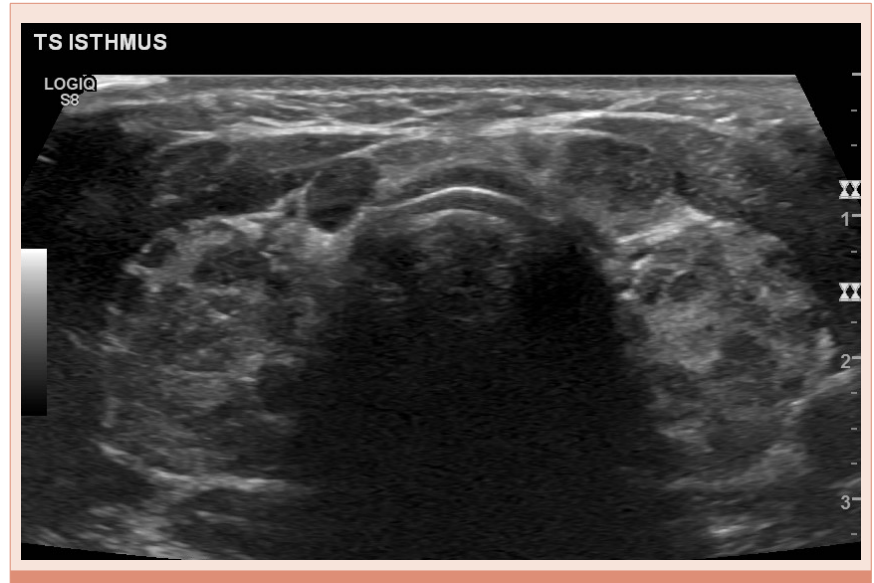

Figure 4: Transverse image of the thyroid gland at six months after the origina presentation to our hospital. The thyroid gland is diffusely hypoechoic and heterogeneous with pseudomicro- and pseudomacronodular appearances, indicating persistence of chronic lymphocytic thyroiditis and no discreet 'true' thyroid nodules. A lymph node can be seen at the border between the right upper thyroid lobe and the isthmus; this can be mistaken for a thyroid nodule. However, on closer examination it was shown to be separate from the thyroid gland and to obey the typical morphology of a reactive (benign) lymph node. 
features or change in the dimensions of a lesion. The relevant time lag may also allow for the inflammation to ameliorate in line with the natural history of the disease. Moreover, evidence exists to suggest that thyroxinee replacement therapy may have a beneficial effect in the clinical course of the disease and the antibody titer [7].

Finally, when thyroid FNA samples are sent for cytology it is prudent to inform the cytopathologist of whether there is underlying thyroiditis as it is not uncommon for thyroid nodules to be misdiagnosed as Thy $3 \mathrm{a}$ and $3 \mathrm{f}$ (Bethesda classification stage 3 and 4 ) in the presence of CLT.

\section{Conclusion}

In summary, reactive lymph nodes in CLT are common and can be mistaken with thyroid nodules because of their close proximity with the thyroid gland.

\section{References}

1. Perros P, Boelaert K, Colley S, Evans C, Evans RM, et al. (2014) Guidelines for the management of thyroid cancer. Clin Endocrinol (Oxf) 81: 1-122.

2. Wong KT, Ahuja AT (2012) Benign thyroid conditions. In: Sofferman RA Ahuja AT, eds. Ultrasound of the thyroid and parathyroid glands. Burlington: Springer 61-106.

3. Lupo MA, Levine RA (2012) Ultrasound of diffuse thyroid enlargement. In: Baskin JH, Duick DS, Levine RA, eds. Thyroid ultrasound and ultrasoundguided FNA. Salt Lake City: Springer 99-125.

4. Bonavita JA, Mayo J, Babb J, Bennett G, Oweity T, et al. (2009) Pattern recognition of benign nodules at ultrasound of the thyroid: which nodules can be left alone? AJR Am J Roentgenol 193: 207-213.

5. Jones MR, Mohamed H, Catlin J, April D, Al-Qurayshi Z, et al. (2015) The presentation of lymph nodes in Hashimoto's thyroiditis on ultrasound. Gland Surg 4: 301-306.

6. Serres-Créixams X, Castells-Fusté I, Pruna-Comella X, Yetano-Laguna V, Garriga-Farriol V, et al. (2008) Paratracheal lymph nodes: a new sonographic finding in autoimmune thyroiditis. J Clin Ultrasound 36: 418-421.

7. Özen S1, Berk Ö, Şimşek DG, Darcan S (2011) Clinical course of Hashimoto's thyroiditis and effects of levothyroxinee therapy on the clinical course of the disease in children and adolescents. J Clin Res Pediatr Endocrinol 3: 192 197. 